\title{
Detection, Localization, and Tracking of Unauthorized UAS and Jammers
}

\author{
İsmail Güvenç ${ }^{1}$, Ozgur Ozdemir ${ }^{1}$, Yavuz Yapici ${ }^{1}$, Hani Mehrpouyan ${ }^{2}$, and David Matolak ${ }^{3}$ \\ ${ }^{1}$ Department of Electrical and Computer Engineering, North Carolina State University, Raleigh, NC, USA \\ ${ }^{2}$ Department of Electrical and Computer Engineering, Boise State University, Boise, ID, USA \\ ${ }^{3}$ Department of Electrical Engineering, University of South Carolina, Columbia, SC, USA \\ Email: \{iguvenc, oozdemi, yyapici\}@ncsu.edu, hanimehrpouyan@boisestate.edu, matolak@cec.sc.edu
}

\begin{abstract}
Small unmanned aircraft systems (UASs) are expected to take major roles in future smart cities, for example, by delivering goods and merchandise, potentially serving as mobile hot spots for broadband wireless access, and maintaining surveillance and security. Although they can be used for the betterment of the society, they can also be used by malicious entities to conduct physical and cyber attacks to infrastructure, private/public property, and people. Even for legitimate use-cases of small UASs, air traffic management (ATM) for UASs becomes of critical importance for maintaining safe and collusion-free operation. Therefore, various ways to detect, track, and interdict potentially unauthorized drones carries critical importance for surveillance and ATM applications. In this paper, we will review techniques that rely on ambient radio frequency signals (emitted from UASs), radars, acoustic sensors, and computer vision techniques for detection of malicious UASs. We will present some early experimental and simulation results on radar-based range estimation of UASs, and receding horizon tracking of UASs. Subsequently, we will overview common techniques that are considered for interdiction of UASs.
\end{abstract}

Index Terms-Drones detection, interdiction, Internet of Things (IoT), mmWave radar, surveillance, tracking, UAS, UAV.

\section{INTRODUCTION}

Enabled by the advances and miniaturization in computing, communication, and sensing, unmanned aircraft systems (UASs) such as quadcopters, fixed wing aircrafts, and baloons have found several applications in the past decade. Some of the popular UAS applications include border surveillance [1], agriculture [2], disaster monitoring [3], [4], traffic monitoring [5], remote sensing [6], construction management [7], transportation [8], [9], and telecommunications [10], [11]. Due to their extensive use cases, potential for disruption, and decreasing costs of ownership/operation, UASs have a rapidly growing user community [8], [12]-[22].

In parallel to these advancements, there has been a continued growth in air traffic density and complexity in the United States and across the world. Proliferation of commercial UASs will bring the challenge of integrating the UASs to the national airspace. Even though UASs will initially fly in airspace separate from the piloted aircraft, "mixed airspaces" will be observed in the future. In fact, NASA Aeronautics Research Mission Directorate's (ARMD's) Strategic Implementation Plan (SIP) for years 2025-2035 and beyond 2035 time frames envision a (i) fully integrated terminal, enroute, surface and and arrivals/departures operations (ATM+2), and (ii) fully

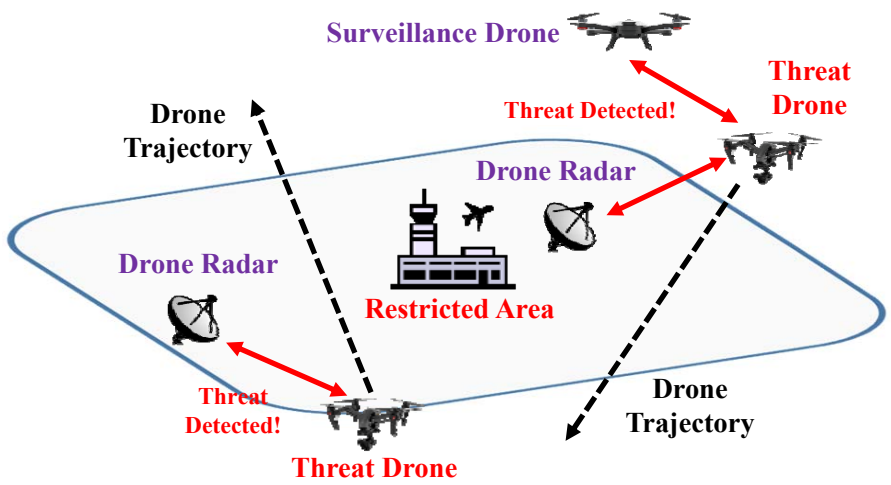

Fig. 1: Detection of unauthorized small UASs around restricted areas.

autonomous trajectory services $(A T M+3)$ for the national airspace [23]. Therefore, in the long term, full integration of UAS throughout the worldwide airspace (and beyond, e.g., stratospheric flights) are expected, which requires effective and safe air traffic management (ATM) techniques for the mixed manned/unmanned flights. This requires development and integration of highly-reliable and efficient communications, navigation, and surveillance technologies among aircraft, ground stations, and other entities.

Safety and efficiency of future ATM relies on adapting to major emerging challenges that small UASs pose. Small UAS have developed to the point that they can carry several pound payloads automatically to any destination within ranges up to 10 miles, at the push of a button, out of the box [24]. They are cheap, disposable and hard to detect and track. Future ATM must safely coexist with a high density of independent small UAS while being able to prevent intruders from endangering the security of protected zones. Therefore, reliable and consistent detection of unauthorized UASs enables timely deployment of countermeasures, reducing or eliminating the potential risks associated with a possible intrusion. Indeed, this challenge has received extensive attention from both academia and industry. For example, some of the recent start-up companies that aim to develop technologies for detecting/tracking commercial UASs include Drone Detect [25], Dedrone [26], Drone Watcher [27], Sky Droner [28], and Sky Safe [29].

In this paper, our main goal is to provide a unified 
review of the available techniques for detecting, tracking, and interdicting small unauthorized UASs around restricted areas, as illustrated in Fig. 1. We consider that UAS detectors are deployed around the area of interest; the detectors can be ground or aerial units (see e.g., the Aerial Dragnet concept from DARPA [30]). If the trajectory of the UAS remains outside the protected area, it is considered to be safe. If the trajectory crosses in the protected area the drone needs to be detected and brought down outside of the protected zone. The UAS detectors can use a variety of techniques: radar (mmWave, UWB, other), radio frequency (RF) signals radiated from UASs, acoustic signals, and computer vision methods. After a review of these techniques and some related experimental results, we will present an approach for tracking of malicious UASs by other UASs.

This paper is part of four review papers that will be presented during the IEEE DASC 2017 special session titled Hyper-Spectral Communications and Networking for ATM (HSCNA). The special session will review the major goals of the recently awarded NASA University Leadership Initiative (ULI) project described in [31]. The project aims to address six major technical challenges related to communications, navigation, and surveillance (CNS) for civil aviation applications. This survey paper will address the technical challenge five in [31] related to unauthorized UAS/jammer detection/localization techniques, and the other five technical challenges are described/reviewed in the other three papers to be presented in the same IEEE DASC special session [32]-[34].

The rest of the paper is organized as follows. In Section II] review of radar based techniques for detection/tracking/classification of drones is presented. In particular, mmWave radars, UWB radars, and other radar techniques are studied. In Section III, drone detection techniques other than radar systems are surveyed; namely, RF based techniques, acoustic sensors, camera vision, and sensor fusion. Section IV provides a review of drone tracking and jammer localization techniques and provides some preliminary results. Finally, Section $\mathrm{V}$ provides some concluding remarks.

\section{RADAR BASED TECHNIQUES}

When there is no active RF radiation from a drone (e.g., fully or mostly autonomous operation), or if such RF signals can not be detected reliably, use of RF radar techniques can be an appealing approach for detecting/tracking drones. As opposed to e.g., optical sensors, radars are active sensors, and they can operate at day or night. In this section, we will consider radar based techniques for detection/tracking/classification of drones and provide a survey of the related work in the literature. In particular, we will study three major categories: mmWave radars see e.g. Fig. 3(a), UWB radars (see e.g. Fig. 3.b)), and other radar based techniques.

The two use case scenarios for radar based drone surveillance are summarized in Fig. 2 (see e.g. [35]). In one use case, Fig. 2(a) shows that one (or more) radars can be used to monitor a restricted area and detect/track drones entering/within that area. In another use case shown in Fig. 2(b), the multiple radars with directional transmissions (e.g., for the purpose of covering a larger area) may also be

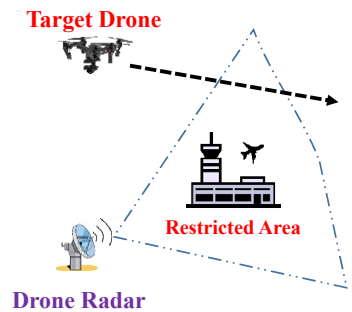

(a) Monitoring of Restricted Area

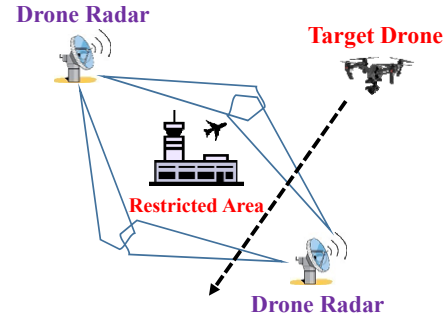

(b) Geofencing of Restricted Area
Fig. 2: Radars for (a) restricted area monitoring, and (b) geofencing.
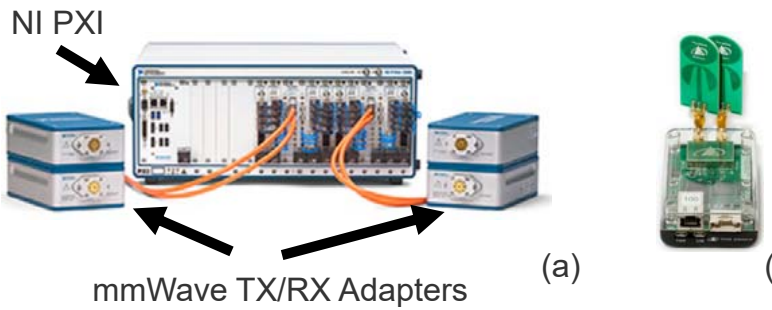

(a)

Fig. 3: (a) mmWave software defined radios (SDRs) from National Instruments [36], and (b) P440 UWB transceiver from Time Domain, Inc. 37|.

used as a geofence, to detect drones entering a restricted area, which may trigger further action for the interdiction of drones.

\section{A. mmWave Radar}

The mmWave radars have very large bandwidths, and hence can provide a high resolution that can facilitate accurate detection, classification, and tracking of unauthorized UASs. A major challenge with radar based detection of micro-drones is that they have very small radar cross section (RCS), and they fly at lower altitudes and at lower speeds compared to larger drones [38].

Use of mmWave radars enables one to analyze precisely the Doppler spectrum, which can provide information for classifying the type of the drone. In particular, the relative motion of the rotating blades of the UASs with respect to bulk motion of the UAS generates time-varying Doppler characteristics (referred as micro-Doppler, see [39] for a nice survey), which may enable highly accurate UAS classification [40], [41]. Even though it is usually difficult to distinguish small UASs from birds using conventional features of the target, revealing the micro-Doppler characteristics enable successful identification even for small-size drones [42]. Especially when the target is moving tangentially with respect to the radar, where the relative distance does not change significantly, the Doppler characteristics are the only source in target classification and even tracking [43]. In addition, the detection and classification of the flying UASs have significant importance for other UASs trying to avoid from possible collisions [44].

In [35], frequency-modulated continuous-wave (FMCW) radar operating at $35 \mathrm{GHz}$ was used to classify DJI Phantom 2 (quadcopter) and DJI S1000 (octocopter) drones, at radar 


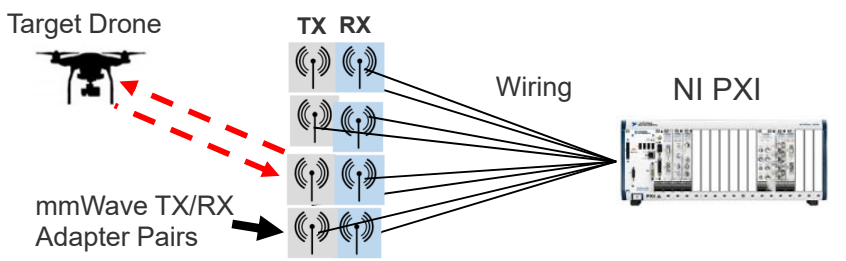

Fig. 4: SDR-based multi-antenna mmWave drone radar.

ranges between $30-90 \mathrm{~m}$. Authors observed wide Doppler sidelobes in range-Doppler profiles due to the high carrier frequency. On the other hand, information about the drone's range, radial velocity, size, type, and even shape can be extracted (and altitude if two antennas are used).

Authors in [45] present a mmWave radar operating at $94 \mathrm{GHz}$ at $20 \mathrm{dBm}$ transmit power, a bandwidth of $1 \mathrm{GHz}$, and a range resolution of $15 \mathrm{~cm}$. The radar can cover distances from $10 \mathrm{~m}$ up to several hundred meters. In [46], the authors considered the use of a 16 receive, 16 transmit MIMO radar to generate 256 virtual elements during signal processing to detect and track drones. The radar operates at $36 \mathrm{GHz}$ central frequency, and uses pulses of duration 500 ns. A hypothesis testing framework is developed to decide whether a drone exists within a certain range/angular cell. Experimental results by the authors show that the target drone can be tracked accurately at distances exceeding $100 \mathrm{~m}$, and at radial velocity of about $5 \mathrm{~m} / \mathrm{s}$.

As a type of multistatic radar, MIMO radar can be considered as a fruitful concept at the intersection of the communication and radar systems. With the use of widely separated antennas, MIMO radar can achieve a high spatial diversity enabling the detection and parameter estimation of the targets more accurately [47]. In this regard, MIMO radar is discussed to achieve a larger diversity gain as compared to the conventional phased array radar, which inherently improves detection and tracking quality [48]. In MIMO radars, non-orthogonal waveforms can be employed for transmission, where a properly chosen set of such waveforms can achieve the same diversity gain as orthogonal waveforms [49]. From a different perspective, MIMO radar can achieve almost constant SNR by statistically averaging over many decorrelated subchannel outputs, where small RCS fluctuations modeled as Swerling I are improved to be of Swerling II case without any loss of time [50]. In [51], a MIMO radar approach is considered, which has been relatively recently developed to thin a radar array. The paper designs mmWave planar arrays for MIMO radar, under the limitation of spreading sequence length due to increased Doppler effects at mmWave frequencies. Both $24 \mathrm{GHz}$ and $94 \mathrm{GHz}$ are considered, and unit cell layouts for the transmitter/receiver array configurations are provided.

Building on these recent related work, we plan to develop an SDR-based mmWave radar testbed to detect/track/classify commercial small UASs. An advantage of such a platform is that it can also be used for other purposes such as mmWave communications and channel sounding. We will develop the testbed using the PXI platform from National Instruments [36] as shown in Fig. 3 (a). Multiple mmWave transceiver/receiver pairs will be integrated into the SDR platform to evaluate

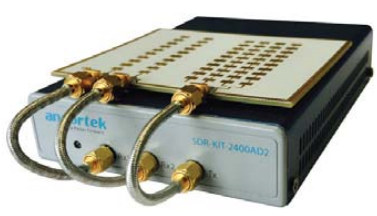

(a) SDR-KIT 2400AD2 K-Band SDR Evaluation Kit (Ancortek)

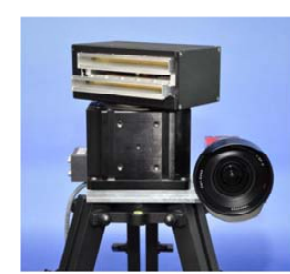

(b) Scanning Surveillance Radar System (Fraunhofer Institute)
Fig. 5: mmWave radar kits from (a) Ancortek [52] and (b) Fraunhofer Institute [53] for drone detection applications.

diversity gain, as shown in Fig. 4 .

The SDRs produced by Ancortec Inc. [52] are smart alternatives for building mmWave radar to detect and track small-size drones. In particular, 2400AD2 SDR kit shown in Fig. 5(a) that operates at the $24-26 \mathrm{GHz}$ band outshines the others with its dual receiver channels enabling interferometry-based micro-Doppler measurements. The prototype by Fraunhofer shown in Fig. 5(b) consists of a mechanically-scanning surveillance radar and a monopulse tracking radar, both of which operate at the $94 \mathrm{GHz}$ centre frequency with $100 \mathrm{~mW}$ transmit power [53]. The system is also supported by a high resolution optical camera to provide LOS vision. The tracking radar emits a $\mathrm{CW}$ signal of $1 \mathrm{GHz}$ bandwidth, and hence, achieves a range resolution as small as $15 \mathrm{~cm}$, which is suitable for small-size drone tracking.

\section{B. UWB Radar}

Due to their very high temporal resolution, UWB technology has been extensively considered in radar applications in the literature, for tracking people (also beyond walls [54]), vehicles [55], [56], heartbeat [57], and vital signs [58]. Flexible FCC regulations for UWB transmissions make the use of UWB radars a viable alternative for detecting/tracking drones.

There are different emission masks defined by the FCC applicable to different types of UWB transmissions: communications devices (separate masks for indoor and outdoor), imaging devices, and vehicular radar systems. The maximum transmit power allowed for UWB devices based on FCC's Part 15 rules is $-41.3 \mathrm{dBm} / \mathrm{MHz}$; however, this maximum transmit power is allowed only within certain range of frequencies, and maximum transmit power limit is lower at other spectrum. In particular, $-41.3 \mathrm{dBm}$ is allowed between $3.1 \mathrm{GHz}$ to $10.6 \mathrm{GHz}$ for communication applications, 1.99 $\mathrm{GHz}$ to $10.6 \mathrm{GHz}$ for through wall imaging applications (limited to law enforcement and rescue teams), and $22 \mathrm{GHz}$ to $29 \mathrm{GHz}$ for vehicular radar systems [59, Ch. 1]. For vehicular radar applications, the UWB radar can be mounted on terrestrial transportation vehicles, and can be actively used while the devices are mobile or stationary [59, Ch. 1].

Use of UWB technology for detection of drones has been considered in the existing literature. In one of the earlier works [60], use of C-band UWB radar (500 MHz bandwidth centered at $6.35 \mathrm{GHz}$ ) at micro air vehicles is considered, with applications including detection of nearby obstacles with small RCS for collusion avoidance. In [61], considering the 


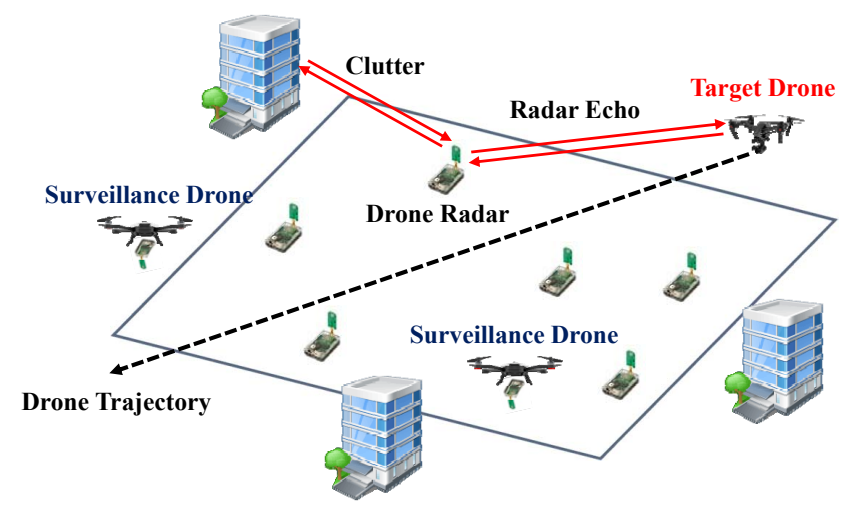

Fig. 6: Distributed ground/airborne UWB radar for drone detection.

24-26 GHz band, characteristics of UWB radar echoes from a drone are studied, which shows that echoes from the drone's rotor blades can be observed for UAS classification purposes. Inverse synthetic aperture radar (ISAR) based imaging of rotating blades in a drone using a Time Domain P410 UWB radar is studied in [62].

In [63] detection of drones using Ku-band $(11.7-12.7 \mathrm{GHz}$ for downlink, $14-14.5 \mathrm{GHz}$ for uplink) battlefield radar is evaluated. In particular, the radar range for a given target detected by the UWB radar is defined as [63], [64]

$$
R_{\max }=\left(\frac{P_{\mathrm{T}} G^{2} \lambda^{2}}{(4 \pi)^{3} F k T_{0} B L} \frac{\sigma}{\rho}\right)^{\frac{1}{4}},
$$

where $P_{\mathrm{T}}$ is the radar transmit power, $G$ is the transmit antenna gain, $\lambda$ denotes the wavelength of the radar signal, $\sigma$ is the RCS of the target object, $F$ denotes the noise figure of radar receiver, $k$ and $T_{0}$ are the Boltzman constant and the temperature, respectively, $B$ is the radar receiver's bandwidth, $L$ captures the losses from the transmission, and finally $\rho$ is the signal-to-noise ratio (SNR) at the radar receiver. Moreover, [63] also shows that the SNR can be expressed as a function of radar's target detection probability $\left(P_{\mathrm{D}}\right)$ and false alarm probability $\left(P_{\mathrm{Fa}}\right)$ as $\rho=\frac{\ln \left(P_{\mathrm{FA}}\right)}{\ln \left(P_{\mathrm{D}}\right)}-1$. Using this relationship with (1), for a fixed $P_{\mathrm{Fa}}$, one can express the detection probability of the target as a function of detection range and different RCS values of the target. The paper then compares the detection probabilities of three commercial small UASs for different values of $P_{\mathrm{Fa}}: \mathrm{X} 8$, High On, and Iris+, resulting in a conclusion that detection probability of Iris + is significantly lower due to its smaller RCS.

In [65], a detect-and-avoid (DAA) UWB radar on-board implementation is presented, which also implements MIMO radar to improve UAS target detection probability. Simulation results with five target drones show that they can be accurately localized in the elevation/azimuth angular domain using the proposed UWB MIMO radar technique. In [66], detection/localization of high-speed moving targets is studied using a short-range UWB radar with an antenna array. In addition to studies that consider using UWB radar for detecting drones, there are also recent literature which consider the use of UWB technology for localization of drones. For example,

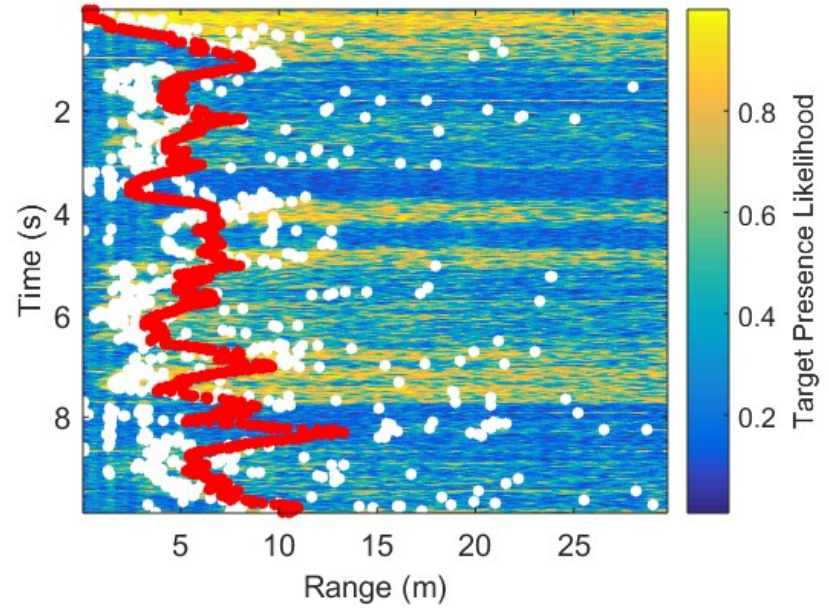

Fig. 7: Range estimation to a UAS using UWB radar [31].

in [67], Time Domain PulseON 400 UWB transceivers at a drone and a ground station are used to estimate the range from the drone. The considered approach uses the two-way time-of-flight technique and can work at communication ranges up to $80 \mathrm{~m}$ for 3D localization of the drone.

In our work, we consider a scenario as in Fig. 6 which consider a distributed UWB radar scenario, with both ground-based and airborne Time Domain P440 UWB radars. We will first consider line-of-sight (LOS) scenarios, and jointly process the data collected at multiple UWB radars for detection/tracking/classification of a target drone.

For proof of concept purposes, we conducted preliminary experiments with the Time Domain UWB radar equipment to evaluate range estimation accuracy to a small quadcopter. A large number of channel impulse response (CIR) realizations based on the reflected radar signals from the UAS were captured at the UWB radar receiver, and consecutive channel impulse responses are subtracted from each other to filter out the multipath components from static objects (clutter). From these data, statistics (e.g., variance and mean) of the Doppler effect can be calculated, which can then be used to find likelihood functions and subsequently the range estimates at each time instant using a maximum likelihood estimator. Finally, a Kalman filter was used to track the range of the target UAS as shown by the red curve in Fig. 7, where the white dots illustrate the instantaneous range estimates to the target drone. Results show that with time domain Kalman filtering the noisy range likelihood estimates can be smoothed to obtain an accurate range estimate to the target UAS. Range estimates at multiple radar locations can be used to detect the UAS's location. Directional antennas and beamforming techniques can be further used to increase the radar detection range.

\section{Other Radar Techniques}

In addition to mmWave and UWB based radar techniques, narrowband radars at lower central frequencies may also be utilized for detection of UASs. For example, in [38], authors consider the use of multi-static radars operating at $2.4 \mathrm{GHz}$ 
to analyze micro-Doppler signatures of micro-drones (DJI Phantom Vision 2) with different payloads, and classify drones into no payload, $200 \mathrm{~g}$ payload, and $500 \mathrm{~g}$ payload categories. A transmit power of $23 \mathrm{dBm}$ was used, along with horizontally polarized antennas with $24 \mathrm{dBi}$ gain and $10^{\circ} \times 10^{\circ}$ beamwidth, at radar to target distances less than $100 \mathrm{~m}$. The proposed feature detection techniques consistently achieve more than $90 \%$ classification accuracy for different payload types.

In a similar context to [38], in [68] authors consider classification of drones with different payloads using a $2.4 \mathrm{GHz}$ radar. Features of the drone related to the RCS and micro-Doppler signatures are used, and classification techniques such as discriminant analysis, naive Bayes, and random forest theory are utilized. In [69], both time domain and micro-Doppler discrimination of the signatures are utilized to detect drones with a $2.4 \mathrm{GHz}$ radar (using a constant false alarm rate detector), by enhancing the difference between the ground clutter and reflections from the drone. Moreover, an extended Kalman filter is introduced for tracking the drone in two dimensions. The RCS simulations and measurement of a DJI drone at a frequency of $2.4 \mathrm{GHz}$ and a bandwidth of $45 \mathrm{MHz}$ is carried out in [70], which sheds light for the effect of different drone/blade materials on the signatures observed at a radar system.

\section{NLOS Radar Based Drone Detection and Tracking}

The detection and tracking of small drones flying at moderate altitudes can be even more problematic in urban areas, as illustrated in Fig. 8 The total obstruction or shadowing imposed by the densely located buildings generally makes even the detection problem very difficult. In urban areas, since it is hard to provide a clean view for LOS transmission towards the target to be detected and tracked, we need to rely on multipaths arising from scattering of the transmitted signal from various obstacles in the environment [71], [72]. This non-LOS (NLOS) based approach, which can also be referred to as "seeing beyond the corner" [73] or "around-the-corner radar" [74], can take the full advantage of multipaths to increase the coverage for detection and tracking functionalities in dense urban areas [75].

Even though the multiple paths have been considered traditionally as nuisance to be eliminated, it becomes a source of spatial diversity by this NLOS based positioning and ranging approaches [71]. In order to coherently combine the multiple signals coming from various paths and extract the range information, some prior information on the topology of the environment, such as exact locations of roads and buildings, should be utilized [76]. In this regard, the channel impulse response or equivalently the scattering map of the environment should be characterized a priori [77]. It can be argued that the detection of a breathing human by micro-Doppler techniques can be extended to NLOS environments when a perfect knowledge of the surrounding geometry is known and multipath signals are employed in ranging [73], [78]. Note that, multiple antennas can also be employed to improve the accuracy and the resolution of this NLOS based ranging approach [79], similar to employing multiple transceivers in synthetic aperture radar (SAR) imaging relying on multipaths $[80]$. Finally, as many transmission

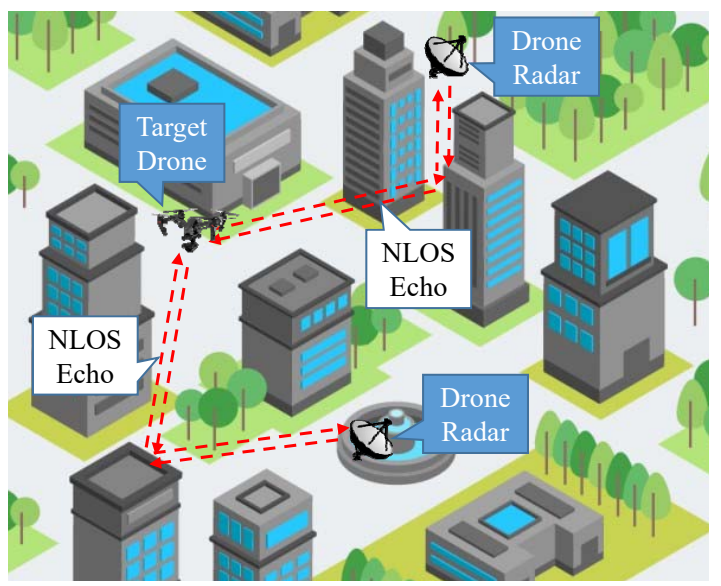

Fig. 8: Detection/tracking of a drone using NLOS radar.

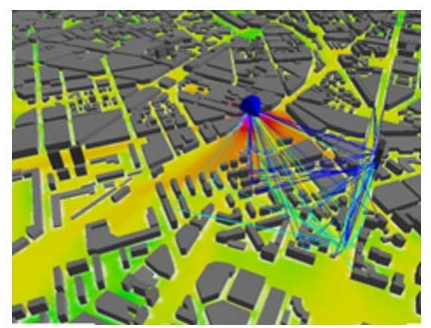

(a) Altair Winprop

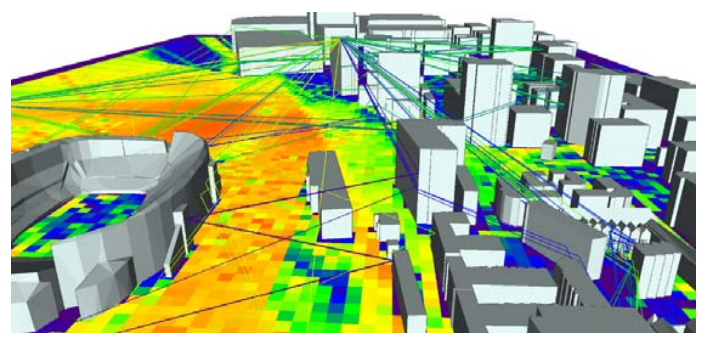

(b) Remcom Wireless Insite

Fig. 9: Ray tracing software: Winprop from Altair [82] and Wireless Insite from Remcom [83].

frequencies for such short-range drone detection applications are also available for communication purposes, joint design of both hardware resources and algorithms for radar and communication systems is of significant importance [81].

\section{E. Ray Tracing Simulations}

Due to challenges involved in measurements for radar based detection of UASs, ray tracing software offers a convenient alternative for simulating different scenarios. Various commercial software tools are available for ray tracing such as WinProp [82] and Wireless InSite [83] as shown in Fig. 9 These software can be used to quickly model urban, suburban, rural, and indoor environments using built in graphical editors. As of the date of this writing, based 


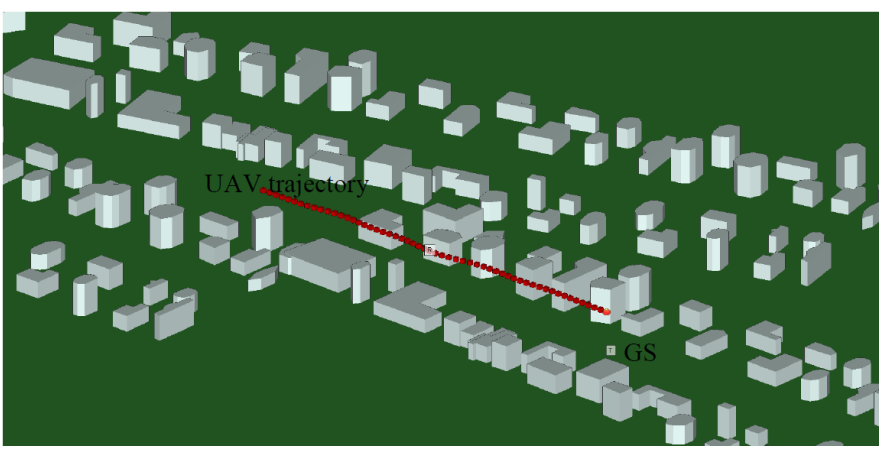

Fig. 10: Simulated urban scenario using Wireless InSite software. Transmitter is located on the ground, and UAS with the on-board receiver follows the illustrated trajectory [84].

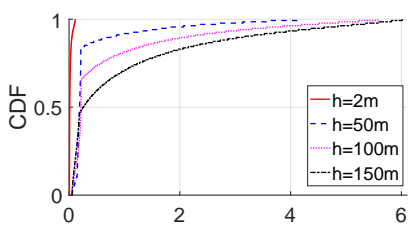

(a) RMS DS (ms)

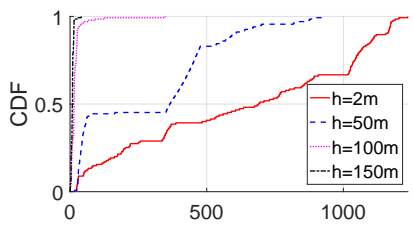

(c) RMS DS (ms)

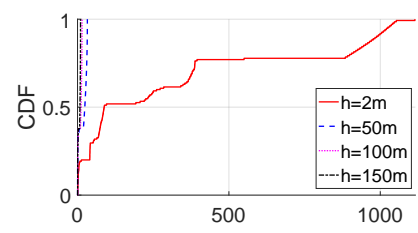

(b) RMS DS (ms)

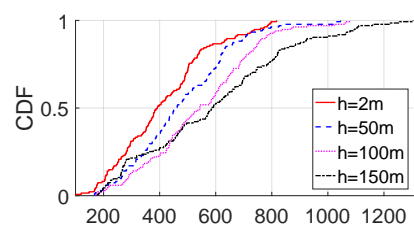

(d) RMS DS (ms)
Fig. 11: Cumulative distribution function (CDF) of the RMS-DS using ray tracing simulations for different UAS heights at $28 \mathrm{GHz}$ mmWave band. Four different environments are considered: (a) Over sea, (b) Rural, (c) Suburban, (d) Urban [84].

on the information in their websites, WinProp supports ray tracing in RF spectrum up to $75 \mathrm{GHz}$, while Wireless Insite supported frequencies up to $100 \mathrm{GHz}$. They also consider realistic propagation phenomena such as the atmospheric oxygen absorption at $60 \mathrm{GHz}$ and diffuse scattering from objects at mmWave bands.

It is possible to model reflections from a moving UAS with ray tracing simulations. However, to the best knowledge of the authors, micro-Doppler effects (e.g., due to signals reflected from the propellers of the drone) can not be characterized using the available ray tracing software in the market. Recent work in the literature show that ray tracing simulations result in more optimistic propagation characteristics when compared to real world measurements in similar environments. For example, results in [85] show that the root mean square (RMS) delay spread (DS) simulations with WinProp in an urban environment are lower when compared to real world measurements in a similar environment, primarily due to diffuse scattering effects in the real world.

Fig. 10 shows the simulation of an urban environment using Wireless InSite [84]. The transmitter is located on the ground, and the UAS with the on-board receiver follows the illustrated path. Considering a similar framework, both radar and communication ray tracing simulations can be conducted for various UAS scenarios and frequencies up to $100 \mathrm{GHz}$. The RMS-DS results obtained using ray tracing simulations for the $28 \mathrm{GHz}$ mmWave band are shown in Fig. 11. The results show that the RMS-DS is the largest for urban environment with high density of scatterers for most of the UAS heights. The RMS-DS increases as a function of the UAS height in the urban environment for the trajectory shown in Fig. 10. The main reason for this behavior is that, at higher UAS altitudes, the UAS moves above the tall buildings, and it can observe signals that are scattered from larger number of surrounding buildings. On the other hand, results in Fig. 11(a), Fig. 11(b), and Fig. 11(c) for over sea, rural, and suburban environments show that, as opposed to the urban environment, the RMS-DS actually decreases with UAS altitude due to less scattering.

\section{OTHER UAS DETECTION APPROACHES}

Apart from radar based techniques, there are other approaches for detecting/tracking drones. In this section, we will study four different alternative approaches: utilization of RF signals radiating from UASs, acoustic sensors, computer vision, and sensor fusion.

\section{A. Radio Frequency Signals from UASs}

One possible approach to detect/track UASs is to use signals that are broadcasted from the UASs. Such signals can be control signals between the UAS and the remote operator (typically at $2.4 \mathrm{GHz}$ ), while they may also be payload signals such as video communications between the UAS and the ground station. In [86], three possible approaches are considered for drone detection: analysis of the reflected signal from the propellers, sniffing the communication between the drone and its controller, and analysis of the reflection patterns from vibration of the drone's body. In [87], frequency hopping spread spectrum signals from a UAS are extracted using SDRs to detect (and jam/spoof) the UAS. This approach can be generalized to train a classifier (e.g., a neural network) for identifying unique RF transmission patterns from popular commercially available UASs. Different RF techniques for remote UAS control are characterized in [88]. In particular, analog FM's RF signature is compared with that of spread spectrum technique, where the latter is shown to have distinguishable RF footprint compared to FM.

\section{B. Acoustic Sensors}

Yet another approach for detecting/classifying UASs is through the use of acoustic sensors [89]-[93], which can detect UASs at distances ranging from from $20 \mathrm{~m}$ [89], [93] up to $600 \mathrm{~m}$ [90]. For example, in [89]-[91] microphone arrays have been used which can (through beamforming) find the direction of the drone in 3D. Hence, using more than one microphone arrays, location of the drone can be identified. In all these three works, a known signature of the drone (which should be captured a-priori) is searched within the real-time spectrogram of the captured acoustic signals. Acoustic detection of a drone can be completely passive and relatively inexpensive; however, they get impacted from factors such as wind and other background noise. 


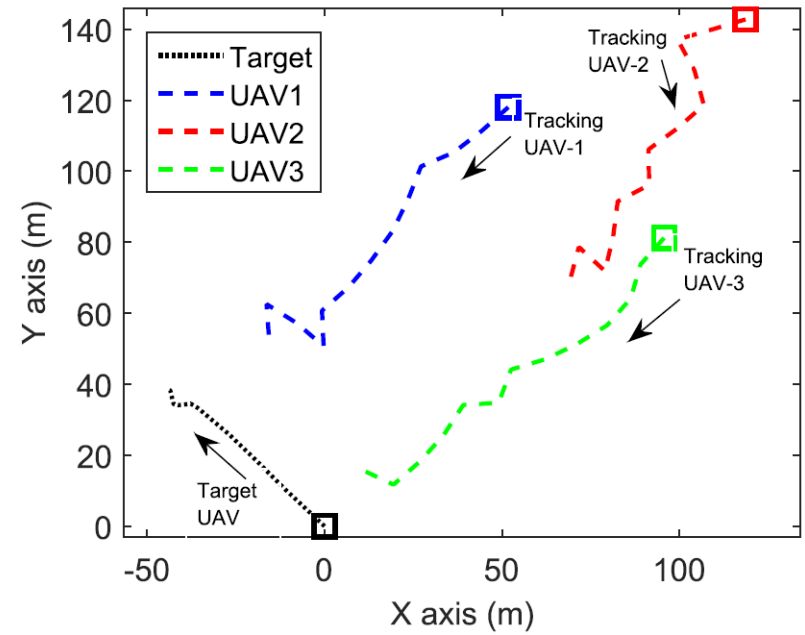

Fig. 12: Target UAS and tracking UAS trajectories [97].

\section{Computer Vision}

Finally, detecting/tracking of unauthorized drones can be achieved using computer vision techniques, which enjoyed extensive advances within the past years [94]. For example, in the ImageNet Large Scale Visual Recognition Competition (ILSVRC), the average detection precision has reached 0.80 in 2016 from 0.22 in 2013. While they suffer from line of sight limitation (e.g., due to cloud, fog, dust), merits of computer vision techniques for drone detection include 1) detecting drones that do not have RF transmission; 2) passive and cheap optical sensors; and 3) inherent directional accuracy. An approach such as in [95] can be used to track a moving and rotating UAS in a complex and dynamic background. Visual/thermal cameras with different fields of views (FOVs) can be used simultaneously for faster and more accurate detection. In [96] a collaborative smart phone sensing based drone detection is studied which e.g. can detect shape, color, orientation of the drones.

\section{Sensor Fusion}

For accurate and quick detection/tracking of UASs, data fusion techniques that may simultaneously use information from multiple different types of sensors can be used. For example, in [45] joint use of a $94 \mathrm{GHz}$ mmWave radar and a high resolution optical camera is considered.

\section{LOCALIZING/TRACKING OF UNAUTHORIZED DRONES AND JAMMERS}

Using the signal strength observations from an unauthorized target drone, it is possible to localize and track them. The signals captured from a drone may be due to legitimate control and/or payload communications from a drone. On the other hand, there may also be drones which are jamming a certain area [97] and they need to be localized and interdicted. To this end, fixed sensors on the ground, as well as one or more other UASs can be used (see Fig. 1).
A specific technical challenge in jammer localization is to locate a low power and smart jammer that can effectively hamper link layer communication reliability by intermittently emitting a small amount of energy relative to emitted signals from authentic sources. The jammer detector should be able to achieve a high probability of detection while maintaining a low probability of false alarm where the jammer is actively avoiding detection. A specific technical challenge in unauthorized UAS detection is to rapidly detect the small size and RCS associated with small UAS. Detection and localization of unauthorized UAS becomes even more challenging when the intrusion is supported by simultaneous jamming. The performance metrics for this work consist of probability of false alarm and probability of detection for jammer and intruder detectors, and localization accuracy for jammer and intruder trackers. We will also study detection/tracking convergence time and computational complexity.

In [97], we have proposed to use multiple UAS to localize and track a mobile UAS jammer as illustrated in Fig. 12 The swarm of UAS aims to achieve the Cramer-Rao lower bound on achievable localization error through maximization of the so-called D-optimality criterion [97], [98]. Authors of [98] used a Fisher information matrix (FIM) predictive model and a receding horizon maximization technique to plan feasible paths for the UAS that lead to the best achievable localization error bound. In other words, the UAS take into account the estimated trajectory of the target while determining their optimum paths. Here we assume that the jammer is transmitting continuously. In practice, the jammer may transmit intermittent signals to minimize its detectability, or only a subset of the transmitted signals by the jammer may be detected by the UAS, e.g., due to frequency hopping transmissions of the target UAS. Some other representative references related to tracking of UASs include [99]-[101]. Building on [97], the intermittent transmission power of the target can be modeled as a Bernoulli random process, which we will use to develop D-optimality criterion and receding horizon tracking techniques for various scenarios, e.g., different propagation channels/frequencies and different jamming strategies. For effective operation, complexity and convergence time are two key metrics.

In [102], we also introduced and experimentally tested a machine-learning based approach for localization of mobile phones using drones, where a random forest algorithm was utilized for finding a mobile users location zone. Intermittently available probe request messages were captured from cellular phones on the ground, and based on the signal strength observations at different UAS locations, location zones of the phones were detected. The proposed approach was able to identify the correct zone of the wireless transmitter with $82 \%$ accuracy. In the proposed research, using various machine learning techniques such as random forest, neural networks, and support vector machines, we will use single and multiple UAS for individually and collaboratively detecting/localizing smart jammers on the ground using signal strength of the jamming signal, where a major goal will be fast detection and localization of the target jammer.

Once an unauthorized drone is identified and localized, different approaches exist for interdicting them. Examples for 


\begin{tabular}{|c|c|}
\hline Detection Technique & References \\
\hline UWB Radar & $[\overline{60}]-\mid \overline{63}],|\overline{65}|-|\overline{67}|$ \\
\hline mmWave Radar & $[35], 445], \mid 46], \mid 52], \mid 53]$ \\
\hline MIMO Radar & $|\overline{46}|-|\overline{51}|$ \\
\hline Other Radar & {$[\overline{38}|,| \overline{68} \mid \overline{-}-\overline{70}]$} \\
\hline NLOS Radar & $\mid \overline{71} \overline{-181} \overline{\mid}$ \\
\hline Ray Tracing Simulators & $82|,| \overline{83} \mid$ \\
\hline Ambient RF Signals & $|\overline{86}|-|\overline{88}|$ \\
\hline Acoustic Signals & $|\overline{89}|-|\overline{93}|$ \\
\hline Computer Vision & $|94|-|96|$ \\
\hline Sensor Fusion & $|\overline{26}|,|\overline{45}|$ \\
\hline Drone Tracking & {$[97],[99]-[101]$} \\
\hline Drone Detection Companies & $25-\mid 29$ \\
\hline one Interdiction & $\overline{103}|-| \overline{10}$ \\
\hline
\end{tabular}

TABLE I: Representative list of references from the literature that are reviewed in this paper.

such approaches include the use of a drone jammer gun [103]; bazooka that fires giant nets to a drone [104]; de-authentication cyber attack to a drone [105]; eagle trained to catch a drone and place in a safe zone [106]; net traps used by large drones to catch smaller drones [107]; and taking control of a drone using GPS spoofing [108].

\section{CONCLUSION}

In this paper we provided an overview of drone detection, localization, classification, and tracking techniques in the literature. A particular emphasis has been on radar based techniques such as mmWave, UWB, $2.4 \mathrm{GHz}, \mathrm{MIMO}$, and NLOS radars for drone detection. Alternative approaches such as those using the RF signals broadcasted from the drones, acoustic and camera vision based methods, and sensor fusion techniques are also discussed. Our future work in the context of a three-year NASA ULI funded project will be implementing and testing several of these approaches using computer simulations and real world experiments. Some of the key references surveyed in this paper are categorized and summarized in Table [.

\section{ACKNOWLEDGEMENT}

This work was supported by the National Aeronautics and Space Administration under Federal Award ID number NNX17AJ94A. The authors would like to thank their project co-investigators Paul Davis and Benjamin Boisevert from Architecture Technology Corporation for their feedback.

\section{REFERENCES}

[1] Z. Sun, P. Wang, M. C. Vuran, M. A. Al-Rodhaan, A. M. Al-Dhelaan, and I. F. Akyildiz, "BorderSense: Border patrol through advanced wireless sensor networks," Ad Hoc Networks, vol. 9, no. 3, pp. 468-477, 2011.

[2] P. Tripicchio, M. Satler, G. Dabisias, E. Ruffaldi, and C. A. Avizzano, "Towards smart farming and sustainable agriculture with drones," in Proc. Int. Conf. Intelligent Environments (IE), 2015, pp. 140-143.

[3] C. Barrado, R. Messeguer, J. López, E. Pastor, E. Santamaria, and P. Royo, "Wildfire monitoring using a mixed air-ground mobile network," IEEE Pervasive Computing, vol. 9, no. 4, pp. 24-32, 2010.

[4] I. Maza, F. Caballero, J. Capitán, J. Martínez-de Dios, and A. Ollero, "Experimental results in multi-UAV coordination for disaster management and civil security applications," J. Intelligent \& Robotic Systems, vol. 61, no. 1-4, pp. 563-585, 2011.
[5] E. Semsch, M. Jakob, D. Pavlicek, and M. Pechoucek, "Autonomous UAV surveillance in complex urban environments," in IEEE Int. Conf. Web Intelligence and Intelligent Agent Technologies (WI-IAT), vol. 2, 2009 , pp. 82-85.

[6] H. Xiang and L. Tian, "Development of a low-cost agricultural remote sensing system based on an autonomous unmanned aerial vehicle (UAV)," Biosystems Engineering, vol. 108, no. 2, pp. 174-190, 2011.

[7] S. Siebert and J. Teizer, "Mobile 3D mapping for surveying earthwork projects using an unmanned aerial vehicle (UAV) system," Elsevier Automation in Construction, vol. 41, pp. 1-14, 2014.

[8] A. Raptopoulos, "No roads? There's a drone for that," June 2013. [Online]. Available: http://www.ted.com/talks/andreas_raptopoulos_ no roads there $s$ a drone for that

[9] “Amazon Prime Air." [Online]. Available: http://www.amazon.com/b? node $=8037720011$

[10] E. Pignaton de Freitas, T. Heimfarth, I. F. Netto, C. E. Lino, C. E. Pereira, A. M. Ferreira, F. R. Wagner, and T. Larsson, "UAV relay network to support WSN connectivity," in Proc. Int. Cong. Ultra Modern Telecommunications and Control Systems and Workshops (ICUMT), 2010, pp. 309-314.

[11] A. Merwaday, A. Tuncer, A. Kumbhar, and I. Guvenc, "Improved throughput coverage in natural disasters: Unmanned aerial base stations for public-safety communications," IEEE Vehicular Technology Magazine, vol. 11, no. 4, pp. 53-60, 2016.

[12] "DIY Drones Website." [Online]. Available: http://diydrones.com/

[13] J. M. Sullivan, "Revolution or evolution? The rise of the UAVs," in Proc. Int. Symp. Technology and Society Weapons and Wires: Prevention and Safety in a Time of Fear (ISTAS), 2005, pp. 94-101.

[14] M. Asadpour, B. Van den Bergh, D. Giustiniano, K. A. Hummel, S. Pollin, and B. Plattner, "Micro aerial vehicle networks: An experimental analysis of challenges and opportunities," IEEE Commun. Mag., pp. 1-11, 2014.

[15] L. Reynaud and T. Rasheed, "Deployable aerial communication networks: challenges for futuristic applications," in Proc. ACM Symp. Performance Eval. Wireless Ad Hoc, Sensor, and Ubiquitous Nnetworks, 2012, pp. 9-16.

[16] R. D'Andrea, "The astounding athletic power of quadcopters," June 2013. [Online]. Available: http://www.ted.com/talks/raffaello_d_ andrea_the_astounding_athletic_power_of_quadcopters

[17] V. Kumar, "Robots that fly ... and cooperate," Feb. 2012. [Online]. Available: http://www.ted.com/talks/vijay_kumar_robots_ that_fly_and_cooperate

[18] L. P. Koh, "A drone's-eye view of conservation," June 2013. [Online]. Available: http://www.ted.com/talks/lian_pin_koh_a_drone_ s_eye_view_of_conservation

[19] R. Dugan, "From mach-20 glider to hummingbird drone," Mar. 2012. [Online]. Available: http://www.ted.com/talks/regina_dugan_ from_mach_20_glider_to_humming_bird_drone

[20] M. Tassey and R. Perkins, "Wireless aerial surveillance platform," DEFCON Conference, Las Vegas, NV, Oct. 2011. [Online]. Available: https://www.youtube.com/watch?v=Iezru0NRZaY

[21] R. Hill, "Phantom network surveillance UAV / Drone," DEFCON Conference, Las Vegas, NV, Aug. 2013. [Online]. Available: https://www.youtube.com/watch?v=i3_DY8Fo5Ps

[22] M. W. Renderman and M. Kershaw, "Build your own UAV 2.0 - wireless mayhem from the heavens!" DEFCON Conference, Las Vegas, NV, Mar. 2012. [Online]. Available: https://www.youtube.com/ watch?v=z0OkXiI 0uI

[23] National Aeronautics and Space Administration, "NASA Aeronautics Strategic Implementation Plan," Read Write, 2015. [Online]. Available: http://www.aeronautics.nasa.gov/strategic-plan.htm

[24] S. Brewster, "Drones Are Getting SmarterAnd More Useful," Read Write, Mar. 2015. [Online]. Available: http://readwrite.com/2015/03/ 19/drones-smarter-more-capable-disaster-relief/

[25] Drone Detector. [Online]. Available: http://www.dronedetector.com/

[26] Dedrone. [Online]. Available: https://www.dedrone.com/en/

[27] Drone Watcher. [Online]. Available: http://www.dronewatcher.com/ 
[28] Sky Droner. [Online]. Available: http://www.skydroner.com/

[29] Sky Safe. [Online]. Available: https://www.skysafe.io/

[30] DARPA Aeriel Dragnet, 2016. [Online]. Available: http://www.darpa. $\mathrm{mil} /$ news-events/aerial-dragnet

[31] D. W. Matolak, I. Guvenc, H. Mehrpouyan, P. Davis, and B. Boisvert, "Hyper-spectral communications, networking and ATM as foundation for safe and efficient future flight: transcending aviation operational limitations with diverse and secure multi-band, multi-mode, and mmWave wireless links," NASA ULI Step B Proposal, Nov. 2016.

[32] D. Matolak, "Hyper-spectral communications, networking and ATM as foundation for safe and efficient future flight: transcending aviation operational limitations with diverse and secure multi-band, multi-mode, and mmWave wireless links: project overview, aviation communications and new signaling," in Proc. AIAA/IEEE Digital Avoinics Systems Conference (DASC), St. Petersburg, FL, Sep. 2017, pp. 1-7.

[33] P. Davis and B. Boisvert, "Hyper-spectral networking concept of operations and future air traffic management simulations," in Proc. AIAA/IEEE Digital Avoinics Systems Conference (DASC), St. Petersburg, FL, Sep. 2017, pp. 1-5.

[34] M. Khatun, H. M. amd D. Matolak, and I. Guvenc, "Millimeter wave systems for airports and short-range aviation communications," in Proc. AIAA/IEEE Digital Avoinics Systems Conference (DASC), St. Petersburg, FL, Sep. 2017, pp. 1-8.

[35] J. Drozdowicz, M. Wielgo, P. Samczynski, K. Kulpa, J. Krzonkalla, M. Mordzonek, M. Bryl, and Z. Jakielaszek, " $35 \mathrm{GHz}$ FMCW drone detection system," in Proc. Int. Radar Symposium (IRS). IEEE, 2016, pp. $1-4$.

[36] National Instruments, "mmWave Transceiver System," Read Write. [Online]. Available: http://www.ni.com/sdr/mmwave/

[37] Time Domain, Inc., "PulsON 440 UWB OEM Module," Read Write. [Online]. Available: http://www.timedomain.com/products/ pulson-440/

[38] F. Fioranelli, M. Ritchie, H. Griffiths, and H. Borrion, "Classification of loaded/unloaded micro-drones using multistatic radar," Electronics Letters, vol. 51, no. 22, pp. 1813-1815, Oct. 2015.

[39] V. C. Chen, "Advances in applications of radar micro-doppler signatures," in Proc. IEEE Conf. Antenna Measurements Applications (CAMA), Nov. 2014, pp. 1-4.

[40] M. Jian, Z. Lu, and V. C. Chen, "Experimental study on radar micro-Doppler signatures of unmanned aerial vehicles," in IEEE Radar Conf., May 2017, pp. 0854-0857.

[41] D. Tahmoush, "Review of micro-Doppler signatures," IET Radar, Sonar Navigation, vol. 9, no. 9, pp. 1140-1146, Dec. 2015.

[42] P. Molchanov, K. Egiazarian, J. Astola, R. I. A. Harmanny, and J. J. M. de Wit, "Classification of small UAVs and birds by micro-Doppler signatures," in European Radar Conf., Oct. 2013, pp. 172-175.

[43] J. A. Nanzer and V. C. Chen, "Microwave interferometric and Doppler radar measurements of a UAV," in IEEE Radar Conf., May 2017, pp. $1628-1633$

[44] M. U. de Haag, C. G. Bartone, and M. S. Braasch, "Flight-test evaluation of small form-factor LiDAR and radar sensors for sUAS detect-and-avoid applications," in IEEE/AIAA Digital Avionics Syst. Conf. (DASC), Sep. 2016, pp. 1-11.

[45] M. Caris, W. Johannes, S. Stanko, and N. Pohl, "Millimeter wave radar for perimeter surveillance and detection of MAVs (Micro Aerial Vehicles)," in Proc. Int. Radar Symposium (IRS), June 2015, pp. 284-287.

[46] J. Klare, O. Biallawons, and D. Cerutti-Maori, "Detection of UAVs using the MIMO radar MIRA-CLE Ka," in Proc. European Conf. Synthetic Aperture Radar, 2016, pp. 1-4.

[47] A. M. Haimovich, R. S. Blum, and L. J. Cimini, "Mimo radar with widely separated antennas," IEEE Trans. Signal Process. Mag., vol. 25, no. 1, pp. 116-129, Jan. 2008.

[48] F. Daum and J. Huang, "MIMO radar: Snake oil or good idea?" in Asilomar Conf. on Signals, Syst. and Comput., Oct. 2008, pp. 183-187.
[49] Q. He and R. S. Blum, "Diversity gain for MIMO neyman-pearson signal detection," IEEE Trans. Signal Process., vol. 59, no. 3, pp. 869-881, Mar. 2011.

[50] E. Fishler, A. Haimovich, R. Blum, R. Cimini, D. Chizhik, and R. Valenzuela, "Performance of MIMO radar systems: Advantages of angular diversity," in Proc. Asilomar Conf. on Signals, Syst. and Comput., vol. 1, Nov. 2004, pp. 305-309 Vol.1.

[51] G. Rankin, A. Tirkel, and A. Leukhin, "Millimeter wave array for UAV imaging MIMO radar," in Proc. IEEE Int. Radar Symposium (IRS), 2015, pp. 499-504.

[52] Ancortek, Inc., "SDR Evaluation Kit," Read Write. [Online]. Available: http://ancortek.com/sdr-kit-2400ad2

[53] M. Caris, W. Johannes, S. Stanko, and N. Pohl, "Millimeter wave radar for perimeter surveillance and detection of MAVs (micro aerial vehicles)," in Proc. Int. Radar Symposium (IRS), 2015, pp. 284-287.

[54] W. Khawaja, F. Koohifar, and I. Guvenc, "UWB radar based beyond wall sensing and tracking for ambient assisted living," in Proc. IEEE Consumer Commun. Netw. Conf. (CCNC), 2017, pp. 142-147.

[55] S. P. Lohmeier, R. Rajaraman, and V. C. Ramasami, "Development of an ultra-wideband radar system for vehicle detection at railway crossings," in Proc. IEEE Conf. Ultra Wideband Systems and Technologies (UWBST), 2002, pp. 207-211.

[56] I. Gresham, A. Jenkins, R. Egri, C. Eswarappa, N. Kinayman, N. Jain, R. Anderson, F. Kolak, R. Wohlert, S. P. Bawell et al., "Ultra-wideband radar sensors for short-range vehicular applications," IEEE Transactions on Microwave Theory and Techniques, vol. 52, no. 9, pp. 2105-2122, 2004.

[57] A. Lazaro, D. Girbau, and R. Villarino, "Analysis of vital signs monitoring using an IR-UWB radar," Progress In Electromagnetics Research, vol. 100, pp. 265-284, 2010.

[58] M. Chia, S. Leong, C. Sim, and K. Chan, "Through-wall UWB radar operating within FCC's mask for sensing heart beat and breathing rate," in Proc. European Radar Conf., 2005, pp. 267-270.

[59] F. Nekoogar, Ultra-wideband communications: fundamentals and applications. Prentice Hall Press, 2005.

[60] R. J. Fontana, E. A. Richley, A. J. Marzullo, L. C. Beard, R. W. Mulloy, and E. Knight, "An ultra wideband radar for micro air vehicle applications," in Proc. IEEE Conf. Ultra Wideband Syst. Technol., 2002, pp. 187-191.

[61] R. Nakamura and H. Hadama, "Characteristics of ultra-wideband radar echoes from a drone," IEICE Communications Express, pp. 1-6, June 2017.

[62] W. Troy, M. Thompson, and Y. Li, "ISAR imaging of rotating blades with an UWB radar," in Proc. Texsas Symp. Wireless and Microwave Circuits and Systems (WMCS), 2015, pp. 1-4.

[63] J. Ochodnickỳ, Z. Matousek, M. Babjak, and J. Kurty, "Drone detection by Ku-band battlefield radar," in Proc. Int. Conf. Military Technologies (ICMT), 2017, pp. 613-616.

[64] Radar Cross Section. [Online]. Available: https: //www.microwaves101.com/encyclopedia/Navy\%20handbook/4. 11\%20Radar\%20Cross-Section\%20(RCS).pdf

[65] Y. A. Nijsure, G. Kaddoum, N. K. Mallat, G. Gagnon, and F. Gagnon, "Cognitive chaotic UWB-MIMO detect-avoid radar for autonomous UAV navigation," IEEE Transactions on Intelligent Transportation Systems, vol. 17, no. 11, pp. 3121-3131, 2016.

[66] G. Wei, Y. Zhou, and S. Wu, "Detection and localization of high speed moving targets using a short-range UWB impulse radar," in Proc. IEEE Radar Conference, 2008, pp. 1-4.

[67] F. Lazzari, A. Buffi, P. Nepa, and S. Lazzari, "Numerical investigation of an UWB localization technique for unmanned aerial vehicles in outdoor scenarios," IEEE Sensors Journal, vol. 17, no. 9, pp. 2896-2903, 2017.

[68] M. Ritchie, F. Fioranelli, H. Borrion, and H. Griffiths, "Multistatic micro-Doppler radar feature extraction for classification of unloaded/loaded micro-drones," IET Radar, Sonar \& Navigation, 2016. 
[69] F. Hoffmann, M. Ritchie, F. Fioranelli, A. Charlish, and H. Griffiths, "Micro-Doppler based detection and tracking of UAVs with multistatic radar," in Proc. IEEE Radar Conf. (RadarConf), 2016, pp. 1-6.

[70] M. Ritchie, F. Fioranelli, H. Griffiths, and B. Torvik, "Micro-drone RCS analysis," in Proc. IEEE Radar Conf., Oct. 2015, pp. 452-456.

[71] P. Chavali and A. Nehorai, "Cognitive radar for target tracking in multipath scenarios," in Proc. Int. Waveform Diversity and Design Conf., Aug. 2010, pp. 110-114.

[72] I. Guvenc and C.-C. Chong, "A survey on TOA based wireless localization and NLOS mitigation techniques," IEEE Communications Surveys \& Tutorials, vol. 11, no. 3, 2009.

[73] A. Sume, M. Gustafsson, A. Janis, S. Nilsson, and A. Orbom, "See-around-corners with coherent radar," in Proc. 20th Radiovetenskap och Kommunikation (RVK), Jun. 2008, pp. 217-221.

[74] K. P. H. Thai, O. Rabaste, J. Bosse, D. Poullin, I. Hinostroza, T. Letertre, and T. Chonavel, "Around-the-corner radar: Detection and localization of a target in non-line of sight," in IEEE Radar Conference (RadarConf), May 2017, pp. 0842-0847.

[75] J. L. Krolik, J. Farrell, and A. Steinhardt, "Exploiting multipath propagation for GMTI in urban environments," in Proc. IEEE Conf. on Radar, Apr. 2006, pp. 65-68.

[76] B. Chakraborty, Y. Li, J. J. Zhang, T. Trueblood, A. Papandreou-Suppappola, and D. Morrell, "Multipath exploitationwith adaptivewaveform design for tracking in urban terrain," in Proc. IEEE Int. Conf. on Acoust., Speech and Signal Process., Mar. 2010, pp. 3894-3897.

[77] H. Luecken and A. Wittneben, "UWB radar imaging based multipath delay prediction for NLOS position estimation," in Proc. IEEE Int. Conf. on Ultra-Wideband (ICUWB), Sep. 2011, pp. 101-105.

[78] M. Gustafsson, A. Andersson, T. Johansson, S. Nilsson, A. Sume, and A. Orbom, "Extraction of human micro-doppler signature in an urban environment using a "sensing-behind-the-corner" radar," IEEE Geosci. Remote Sens. Lett., vol. 13, no. 2, pp. 187-191, Feb. 2016.

[79] L. Li and J. L. Krolik, "Simultaneous target and multipath positioning with MIMO radar," in Proc. IET Int. Conf. on Radar Systems, Oct. 2012, pp. 1-6.

[80] J. F. Nouvel, X. Dupuis, and M. Lesturgie, "Non-line of sight signal analysis: Investigation of interferometry modes over urban area," in Proc. IEEE Asia-Pacific Conf. on SAR (APSAR), Sep. 2015, pp. $582-586$.

[81] M. Scharrenbroich and M. Zatman, "Joint radar-communications resource management," in Proc. IEEE Radar Conf., May 2016, pp. $1-6$.

[82] Awe Communications, "Winprop software suite." [Online]. Available: http://www.awe-communications.com/

[83] Remcom, "Wireles insite propagation software." [Online]. Available: http://www.remcom.com/wireless-insite/

[84] W. Khawaja, O. Ozdemir, and I. Guvenc, "UAV air-to-ground channel characterization for mmWave systems," in Proc. IEEE VTC Fall Workshops, Sep. 2017.

[85] Z. Zhang, J. Ryu, S. Subramanian, and A. Sampath, "Coverage and channel characteristics of millimeter wave band using ray tracing," in Proc. IEEE Int. Conf. Commun. (ICC), June 2015, pp. 1380-1385.

[86] P. Nguyen, M. Ravindranatha, A. Nguyen, R. Han, and T. Vu, "Investigating cost-effective RF-based detection of drones," in Proc. ACM Workshop on Micro Aerial Vehicle Networks, Systems, and Applications for Civilian Use, 2016, pp. 17-22.

[87] Y. Kim, "Security analysis of FHSS-type drone controller," in Proc. Springer Int. Workshop on Information Security Applications, vol. 9503, Jeju Island, Korea, Aug. 2016, p. 240.

[88] M. Haluza and J. Čechák, "Analysis and decoding of radio signals for remote control of drones," in Proc. IEEE New Trends in Signal Processing (NTSP), 2016, pp. 1-5.

[89] G. C. Birch, J. C. Griffin, and M. K. Erdman, "UAS detection, classification, and neutralization: Market survey," Market Survey prepared by Sandia National Laboratories, 2015.
[90] M. Benyamin and G. H. Goldman, "Acoustic Detection and Tracking of a Class I UAS with a Small Tetrahedral Microphone Array," Army Research Laboratory Technical Report (ARL-TR-7086), DTIC Document, Tech. Rep., Sep. 2014.

[91] J. Busset, F. Perrodin, P. Wellig, B. Ott, K. Heutschi, T. Rühl, and T. Nussbaumer, "Detection and tracking of drones using advanced acoustic cameras," in SPIE Security + Defence, vol. 9647, Oct. 2015, pp. $1-8$.

[92] S. Jeon, J.-W. Shin, Y.-J. Lee, W.-H. Kim, Y. Kwon, and H.-Y. Yang, "Empirical study of drone sound detection in real-life environment with deep neural networks," https://arxiv.org/pdf/1701.05779.pdf.

[93] L. Hauzenberger and E. Holmberg Ohlsson, "Drone detection using audio analysis," MS Thesis, June 2015. [Online]. Available: https://lup.lub.lu.se/student-papers/search/publication/7362609

[94] J. Lemley, S. Bazrafkan, and P. Corcoran, "Deep learning for consumer devices and services: Pushing the limits for machine learning, artificial intelligence, and computer vision." IEEE Consumer Electronics Magazine, vol. 6, no. 2, pp. 48-56, April 2017.

[95] G. Nebehay and R. Pflugfelder, "Clustering of Static-Adaptive correspondences for deformable object tracking," in Computer Vision and Pattern Recognition. IEEE, Jun. 2015.

[96] S. K. Boddhu, M. McCartney, O. Ceccopieri, and R. L. Williams, "A collaborative smartphone sensing platform for detecting and tracking hostile drones," SPIE Defense, Security, and Sensing, pp. 874 211-874211, 2013.

[97] F. Koohifar, A. Kumbhar, and I. Guvenc, "Receding horizon Multi-UAV cooperative tracking of moving RF source," IEEE Communications Letters, vol. 21, no. 6, pp. 1433-1436, June 2017.

[98] S. A. A. Shahidian and H. Soltanizadeh, "Path planning for two unmanned aerial vehicles in passive localization of radio sources," Aerospace Science and Technology, vol. 58, pp. 189-196, Nov. 2016.

[99] S. M. M. Dehghan, M. S. Tavakkoli, and H. Moradi, "Path planning for localization of an RF source by multiple UAVs on the Crammer-Rao Lower Bound," in Proc. Int. Conf. Robotics and Mechatronics (ICRoM), 2013, pp. 68-73.

[100] S. M. M. Dehghan, M. Farmani, and H. Moradi, "Aerial localization of an rf source in NLOS condition," in Proc. IEEE Int. Conf. Robotics and Biomimetics (ROBIO), 2012, pp. 1146-1151.

[101] S. M. M. Dehghan, H. Moradi, and S. A. A. Shahidian, "Optimal path planning for DRSSI based localization of an RF source by multiple UAVs," in Proc. Int. Conf. Robotics and Mechatronics (ICRoM), 2014, pp. 558-563.

[102] V. Acuna, A. Kumbhar, E. Vattapparamban, F. Rajabli, and I. Guvenc, "Localization of WiFi devices using probe requests captured at unmanned aerial vehicles," in Proc. IEEE Wireless Commun. Netw. Conf. (WCNC), Mar. 2017, pp. 1-6.

[103] K. Mizokami, "The U.S. Government Is Buying 100 Drone-Jamming Radio Rifles," Popular Mechanics Magazine, May 2016. [Online]. Available: http://www.popularmechanics.com/military/ weapons/a20914/dod-dhs-buying-drone-jammers/

[104] M. Burns, "The SkyWall 100 bazooka captures drones with a giant net," Tech Crunch Magazine, Mar. 2016. [Online]. Available: https://techcrunch.com/2016/03/04/ the-skywall-100-bazooka-captures-drones-with-a-giant-net/

[105] E. Vattapparamban, İ. Güvenç, A. İ. Yurekli, K. Akkaya, and S. Uluağaç, "Drones for smart cities: Issues in cybersecurity, privacy, and public safety," in Proc. IEEE Int. Wireless Communications and Mobile Computing Conf. (IWCMC), Cyprus, Paphos, Sep. 2016, pp. 216-221.

[106] T. Witherow, "Police set to use EAGLES to foil terrorist drone attacks," Daily Mail, Feb. 2016. [Online]. Available: http: //www.dailymail.co.uk/news/article-3436572/

[107] S. Liberatore, "How do you catch a drone? With an even BIGGER drone and a giant net," Daily Mail, Dec. 2015. [Online]. Available: http://www.dailymail.co.uk/sciencetech/article-3356746

[108] A. J. Kerns, D. P. Shepard, J. A. Bhatti, and T. E. Humphreys, "Unmanned aircraft capture and control via GPS spoofing," Journal of Field Robotics, vol. 31, no. 4, pp. 617-636, 2014. 